\title{
An Energy-Efficient Pulse Position Modulation Transmitter for Galvanic Intrabody Communications
}

\author{
MirHojjat Seyedi, Zibo Cai, and Daniel T.H. Lai \\ College of Engineering and Science \\ Victoria University \\ Melbourne, Australia \\ mirhojjat.seyedi@live.vu.edu.au
}

\author{
Francois Rivet \\ University of Bordeaux \\ IMS Laboratory \\ Bordeaux, France \\ francois.rivet@ims-bordeaux.fr
}

\begin{abstract}
Intrabody communications (IBC) is a novel communication technique which uses the human body itself as the signal propagation medium. This communication method is categorized as a physical layer of IEEE 802.15.6 or Wireless Body Area Network (WBAN) standard. It is significant to investigate the IBC systems to improve the transceiver design characteristics such as data rate and power consumption. In this paper, we propose a new IBC transmitter implementing pulse position modulation (PPM) scheme based on impulse radio. A FPGA is employed to implement the architecture of a carrierfree PPM transmission. Results demonstrate the data rate of $1.56 \mathrm{Mb} / \mathrm{s}$ which is suitable for the galvanic coupling IBC method. The PPM transmitter power consumption is $2.0 \mathrm{~mW}$ with $3.3 \mathrm{~V}$ supply voltage. Having energy efficiency as low as $1.28 \mathrm{~nJ} / \mathrm{bit}$ provides an enhanced solution for portable biomedical applications based on body area networks.
\end{abstract}

Keywords-energy-efficient; FPGA; galvanic intrabody communication; pulse position modulation;

\section{INTRODUCTION}

Imagine a world in which simply as you go about your daily life, your medical check-up happens every day. Such systematic monitoring helps to defuse the reliance on medical personnel for current medical disorders like heart attack or stroke. It could prevent frequent hospital visits and save costs for both patient and healthcare system [1]. Nowadays, wireless portable monitoring devices present a revolutionary change in healthcare applications by means of portable devices. WBAN (wireless body area network) is a new ratified communication protocol to standardize the use of wireless techniques in, on, and around the human body. This protocol outlined three physical layer schemes as ultra-wideband (UWB), narrowband (NB), and human body communication (HBC). The humancentric WBAN operation needs to take the technical hardware requirements into account. This raises research issues concerning transceiver circuit design, as a fundamental stage of WBAN system, particularly reducing power consumption while improving the data rate.

Among the WBAN PHYs, HBC is energy efficient compared to both UWB and NB. For instance, the reported energy consumption for an UWB communication system was
$2.5 \mathrm{~nJ} / \mathrm{b}$. While a $0.24 \mathrm{~nJ} / \mathrm{b}$ was indicated for the recent $\mathrm{HBC}$ system [2]. HBC or Intrabody Communication (IBC) is a novel data communication technique using the human body itself as the propagation medium with transmission power below $1.0 \mathrm{~mW}$. IBC can be classified into two basic procedures: Capacitive coupling method and Galvanic coupling method. The former achieved up to $10 \mathrm{Mb} / \mathrm{s}$ data rates in the tens of $\mathrm{MHz}$ frequency bands and the latter approximately $64 \mathrm{~kb} / \mathrm{s}$ for frequencies less than $10 \mathrm{MHz}$ [3]. Depending on different kinds of biomedical signals, i.e. analog or digital, the IBC transceiver may consist of one or more subsystems such as analog-to-digital or digital-to-analog converter (ADC or DAC) [4]. Based on the human body channel limitations and body tissues characteristics in different frequency domain, direct data transmission through the human body lead to a large transmitted voltage or power [5]. Therefore, signals produced by various medical devices are not always suitable for direct transmission over a body channel. Hence a modulator or demodulator should be considered in IBC transceiver design.

The first IBC transceiver was proposed by Zimmerman in 1995 [6]. He employed on-off keying (OOK) modulation scheme in the IBC system due to its simple design and implementation. This preliminary prototype of capacitive coupling IBC indicated data rate and power consumption of $2.4 \mathrm{~kb} / \mathrm{s}$ and $400 \mathrm{~mW}$, respectively. Following several attempts which employed different kinds of analog and digital modulation schemes [7, 8], in 2012, Bae et al. [2] reported the analog IBC transceiver which used a double-FSK (frequency shift keying) modulation scheme which decreased the power consumption of the transceiver to $5.4 \mathrm{~mW}$. Meanwhile, in some of the past studies, the coded digital signal was directly transmitted through the body $[9,10]$. They proposed the digital Manchester data encoding transmitter. Harikomar et al. [10] used a field-programmable gate array (FPGA) to transmit the digital Manchester coded data through the human body without any modulation. Since neither modulation module nor analog to digital converter (ADC) are required in digital transmitter method, the power consumption of their system was $2.0 \mathrm{~mW}$. However, an error in Manchester code signals leads to a huge bit error in entire received signal. The main objective of this paper is to implement the energy efficient 
transmitter i.e. high data throughput as well as low power consumption transmitter for IBC applications. In this work, we propose a digital transmitter based on pulse position modulation scheme which was implemented on FPGA. Invivo experiments had been conducted to evaluate the communication schemes for input digital signal within the galvanic coupling IBC method.

\section{IBC SYSTEM DESIGN}

\section{A. IBC Coupling Method}

IBC can be classified into two fundamental coupling types: capacitive coupling and galvanic coupling method. This segmentation is based on how data is transmitted through the body. Since capacitive coupling has a return path going through the ground, it is strongly influenced by the environment. It is susceptible to external interference such as from power lines $(50 \mathrm{~Hz}$ mains) and even other nearby WBAN devices. However, galvanic coupling decreases dependency on the environment. The current propagation in galvanic coupling takes place within the human tissue and the influence of external interfering signals is negligible. Therefore, the galvanic approach is the preferred method to test the proposed transmitter in this study. Our transmitter demonstrated a higher data rate compared to past galvanic coupling IBC systems.

\section{B. IBC Transmitter Architecture}

The significance of developing an IBC system lies in the vital role that transmitting human physiological parameters has in healthcare monitoring field. Therefore, the IBC transmitter should have acceptable simplicity, high data rate and be energy efficient. We argue that an optimal modulation scheme for IBC would allow the mentioned factors.

In terms of the complexity of different modulation schemes, both OOK and pulse position modulation (PPM) are the potential candidates that allow the implementation of simple transmission circuit. Since PPM is a time based technique, it is more immune to false detection compared to OOK which is a shape-based modulation scheme. Due to similar amplitude pulses of PPM the detection of channel noise is simpler than OOK. Hence more robust and power efficient transmitters are provided by PPM [11].

PPM is a well-known modulation scheme which is widely employed in past UWB WBAN systems [11, 12]. It uses pulse positions to encode the original data signal. The changes in pulse polarities in time variation due to PPM, break the regular intervals in the transmission, and therefore smoothen the overall spectrum by diminishing the line spectrum components [12]. Fig. 1 describes the general structure of the PPM transmitter in IBC method whereas the output of PPM transmitter is directly connected to the human body. Additionally, Fig. 1 indicates that PPM scheme could be implemented without external carrier signal requirement. Carrier-free PPM results in a simplified architecture. Through the analytical expression of the PPM signal, $s(t)$, can be achieved as follows:

$$
s(t)=\sum_{k=0}^{K} p\left(t-b_{k} \frac{T}{2}-k T\right)
$$

where $p$ and $T$ represent the pulse waveform and symbol period, respectively. The values of $b_{k}$ for the $k^{\text {th }}$ transmitted bit are assumed 0 or 1 .

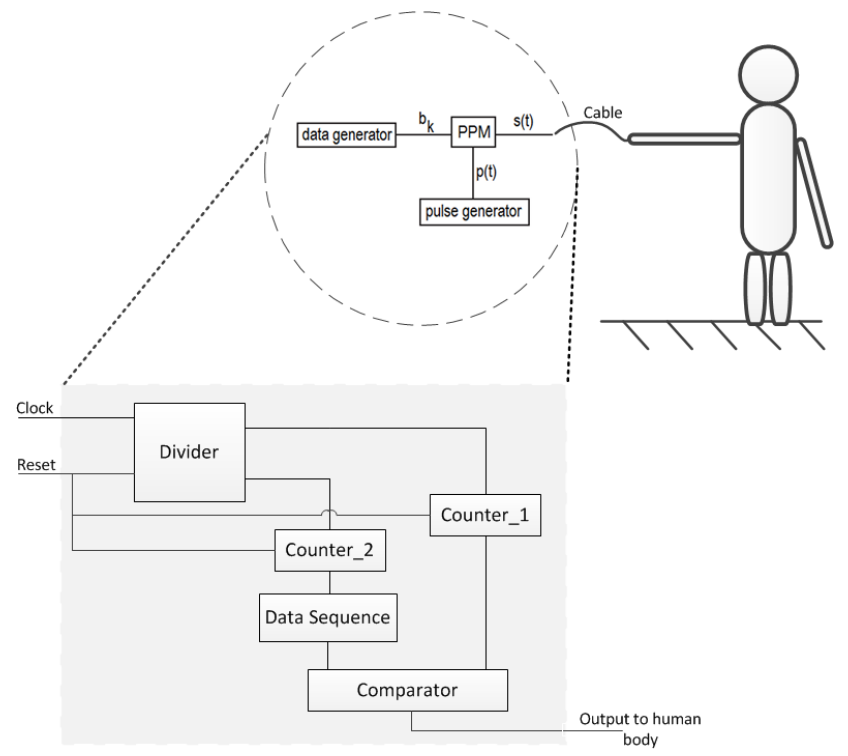

Figure 1. A simplified architecture of the PPM IBC transmitter

\section{The Implementation}

The proposed IBC PPM transmitter architecture is shown in Fig. 1. A FPGA development board is used to implement the whole IBC transmitter circuit. This architecture aims to generate a digital signal which is able to propagate through the human body. Our configurable architecture consists of five main components: a divider, two counters, a data sequence, and a comparator. Since the carrier-free PPM scheme is used in the architecture, the clock frequency of FPGA board is employed as an input pulse. This main clock value is divided to two different clocks by means of divider component. These clocks activate both counter_1 and counter 2. The outputs of counter_1 and data sequence are compared in the comparator and once the output values become equal, the output signal will be ' 1 '. Otherwise the output of ' 0 ' will be generated.

Fig. 2 shows the output of proposed transmitter. It is assumed that 16 slices are transmitted in a time frame. Each time frame is divided into 4 time slots and each time slot contains 4 time slices encoding 4 bits. It is worth noting that for this work, each time slot only 1 slice out of 4 will be transmitted i.e. 4 slices per frame.

In order to generate the mentioned pattern, the FPGA configuration is specified using a hardware description language (HDL). The clock frequency of FPGA is divided by 16 and 64 to run the counters. The position of active slice is selected by programmable data sequence block. 


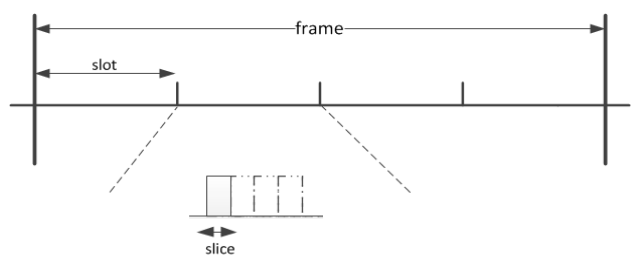

Figure 2. The transmitter output pattern in the proposed IBC system

\section{EMPIRICAL MEASUREMENTS}

\section{A. Measurement setup}

Since the system based on FPGA provides advanced features such as low power and cost, high flexibility, and high data rate values, the design was implemented on FPGA instead of ASIC design. The proposed circuit of IBC transmitter (see Fig. 1) was built on Xilinx Virtex5XC5VLX50T FPGA Genesys board (Digilent Inc., WA). The clock frequency of FPGA board is $100 \mathrm{MHz}$. Also, a $3.3 \mathrm{~V}$ power supply is required to run the transmitter system. In order to produce a real IBC situation and isolate the transmitter from main power effects, a battery was used to run FPGA board. Additionally, to estimate the power consumption of whole transmitter system Xilinx ISE Xpower analyzer tool was used. In measurement setup, an oscilloscope (MSO 5204 Mixed Signal Oscilloscope, Tektronix Inc., OR) was employed to detect the received signal after transmission through the body. A pair of commercial self-adhesive $\mathrm{Ag} / \mathrm{AgCl}$ single electrodes (Noraxon Inc., AZ) was used as transmitter and receiver electrodes. They provide impedance matching in the measurement setup (see Fig. 3).

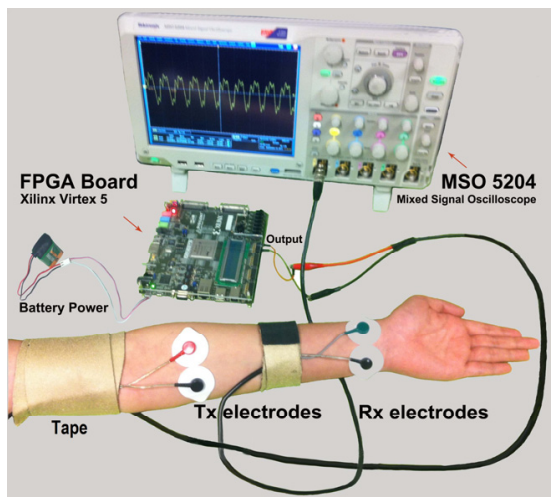

Figure 3. The measurement setup of galvanic coupling IBC using FPGA board, transmitter and receiver electrodes, and oscilloscope

\section{B. Protocol}

In this study, two healthy male volunteers participated in the experiments. The subjects were 30 and 31 years old, 182 and $179 \mathrm{~cm}$ height, and 75 and $67 \mathrm{~kg}$ weight, respectively. The output amplitude of battery-powered FPGA was $0.63 \mathrm{~V}$ which is considered safe for the human body [13].

In all experiments, the transmitter and receiver electrodes were attached on the subject's left forearm. The distance between transmitter and receiver electrodes was $20 \mathrm{~cm}$. The receiver electrodes were close to the wrist and the transmitter electrodes were near the elbow joint (forearm). During experimental conditions inter-electrode distance was set to 3.8 $\mathrm{cm}$. The subjects were told to stand and hold their left arm horizontally (parallel with earth ground) while the arm distance from earth was 138 and $129 \mathrm{~cm}$ for both subjects, respectively. For each experiment setup, the received signal was measured by the oscilloscope. The measurements for each position were repeated three times over three different days and the average data was reported.

\section{RESULTS}

The baseband signal was modulated using PPM to transmit the digital data through the body communications channel. The characteristics of IBC transmitter were measured by means of the oscilloscope. Fig. 4 demonstrates the waveform of a PPM data sequence. The duration of each slot is 640 ns which contains four slices of 160 ns duration. Therefore, the maximum data rate of the transmitter is 1.56 $\mathrm{Mb} / \mathrm{s}$ when one bit is encoded in each time slot. The voltage amplitude of received PPM signal is $0.63 \mathrm{~V}$. Fig. 5 shows the frequency responses of the transmitter output. The results indicate that the effect of noise is insignificant at frequency

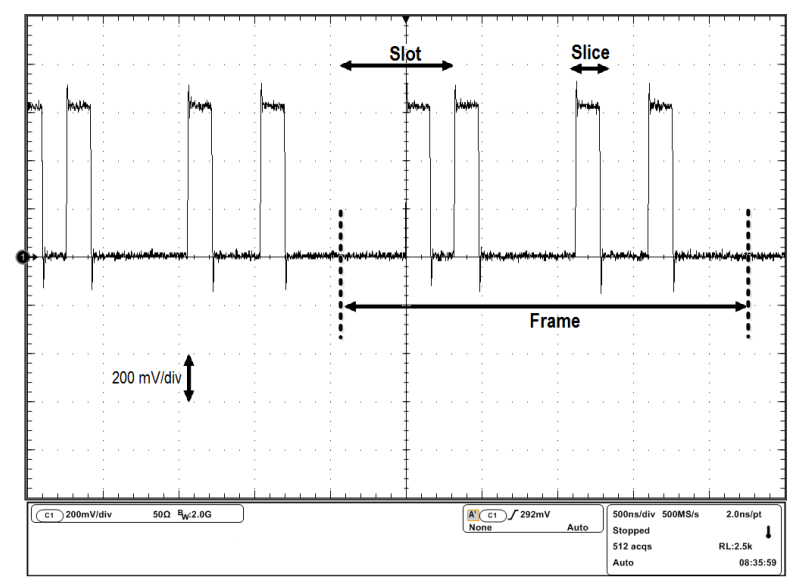

Figure 4. Waveform of PPM transmitter output

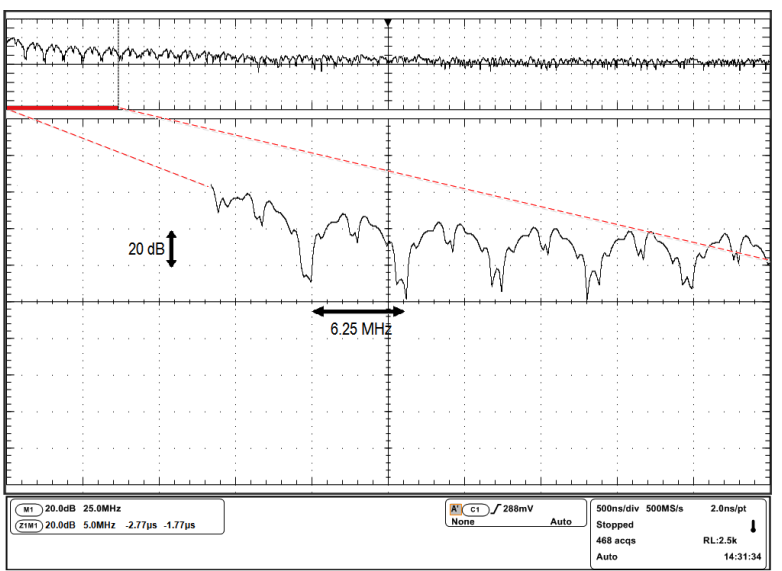

Figure 5. Spectrum measured at PPM transmitter output 


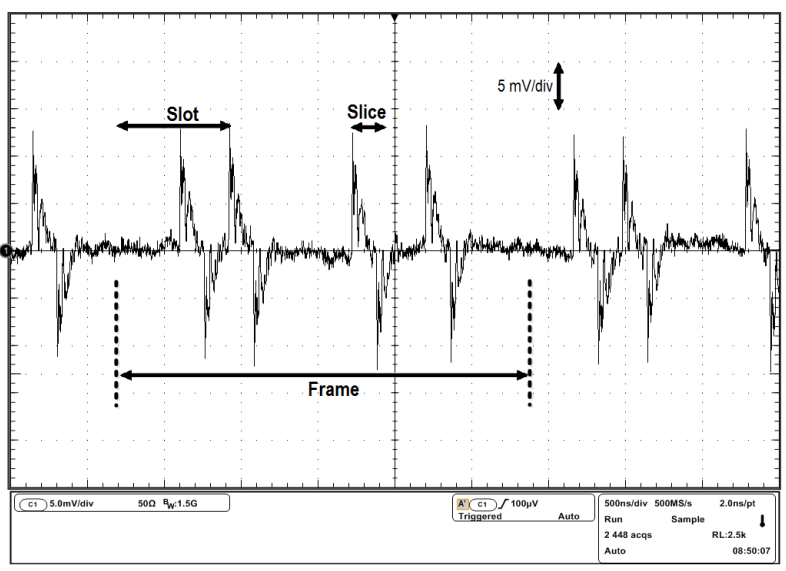

Figure 6. The detected signal from on-body electrodes

range up to $65 \mathrm{MHz}$. Above this frequency, the signal is too noisy to be recognized. Therefore, the output of proposed PPM transmitter is suitable for IBC applications [3]. Fig. 6 shows the received signal from human body which has the amplitude of $13 \mathrm{mV}$. Hence the calculated signal attenuation is around $34 \mathrm{~dB}$. Additionally, the obtained results from second test subject represent the attenuation of $31.5 \mathrm{~dB}$.

Fig. 7 shows the bit error rate (BER) performance for PPM signal transmission using the galvanic coupling IBC method. BER quantifies the reliability of the entire communication system. The PPM BER is mathematically calculated [14] using detected voltage power in receiver electrodes during different distances between transmitter and receiver electrodes.

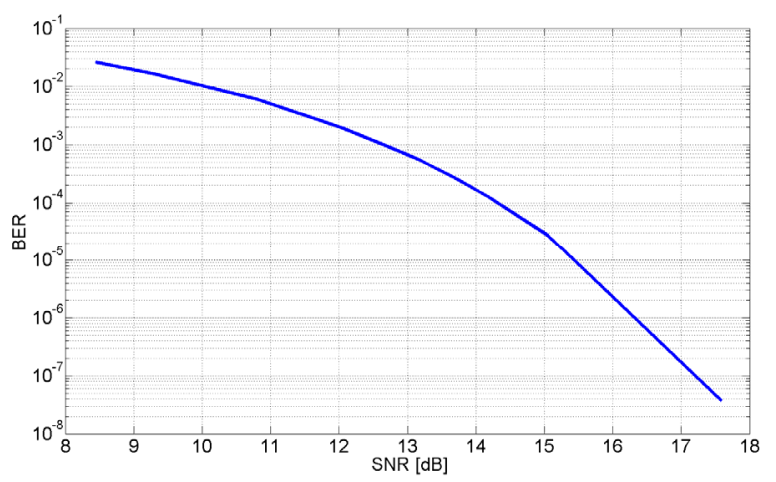

Figure 7. BER Performance of PPM IBC system

Table I, summarizes the performance of our design and compares it to the past galvanic and capacitive coupling IBC systems. Our transmitter consumes only $2.0 \mathrm{~mW}$ energy in data rate of $1.56 \mathrm{Mb} / \mathrm{s}$, compared to the previous galvanic coupling which reported $35 \mathrm{~mW}$ of power consumption [9]. The high data rate of proposed system makes it suitable to be employed in biomedical application [2].

\section{CONCLUSION}

IBC is an efficient data transmission technology for shortrange transmission using human body as the communication channel. This paper focused on a simple transmitter design using PPM modulation for galvanic coupling IBC. It was implemented on battery-powered FPGA board. The low energy consumption per bit $1.28 \mathrm{~nJ} / \mathrm{b}$ suggests that the proposed PPM transmitter is more suitable for portable biomedical devices in the future.

TABLE I. THE CHARACTERISTICS PF IBC TRANSMITTER DESIGN

\begin{tabular}{|c|c|c|c|c|}
\hline Parameters & {$[2]$} & {$[5]$} & {$[8]$} & $\begin{array}{c}\text { This } \\
\text { work }\end{array}$ \\
\hline supply voltage & $1.0 \mathrm{~V}$ & $0.5 \mathrm{~V}$ & $3.3 \mathrm{~V}$ & $3.3 \mathrm{~V}$ \\
\hline $\begin{array}{c}\text { body coupling } \\
\text { method }\end{array}$ & $\begin{array}{c}\text { Capacitive } \\
\text { (ASIC) }\end{array}$ & $\begin{array}{c}\text { Capacitive } \\
\text { (ASIC) }\end{array}$ & $\begin{array}{c}\text { Galvanic } \\
(\text { FPGA })\end{array}$ & $\begin{array}{c}\text { Galvanic } \\
(\text { FPGA })\end{array}$ \\
\hline modulation & FSK double & OOK & $\begin{array}{c}\text { BPSK } \\
\text { QPSK }\end{array}$ & PPM \\
\hline data rate & $1 \mathrm{k}-10 \mathrm{Mb} / \mathrm{s}$ & $2 \mathrm{Mb} / \mathrm{s}$ & $64 \mathrm{~kb} / \mathrm{s}$ & $1.56 \mathrm{Mb} / \mathrm{s}$ \\
\hline $\begin{array}{c}\text { power } \\
\text { consumption }\end{array}$ & $2.0 \mathrm{~mW}$ & $4.5 \mathrm{~mW}$ & $35 \mathrm{~mW}$ & $\begin{array}{c}2.0 \mathrm{~mW} \\
(T X \text { only })\end{array}$ \\
\hline energy/bit & $0.24 \mathrm{~nJ} / \mathrm{b}$ & $2.25 \mathrm{~nJ} / \mathrm{b}$ & $547 \mathrm{~nJ} / \mathrm{b}$ & $1.28 \mathrm{~nJ} / \mathrm{b}$ \\
\hline
\end{tabular}

\section{REFERENCES}

[1] J. M. Smith, "The doctor will see you ALWAYS," IEEE Spectrum, vol. 48, no. 10, pp. 56-62, Oct. 2011.

[2] J. Bae, K. Song, H. Lee, H. Cho, and H.-J. Yoo, "A low-energy cristalless double-FSK sensor node transceiver for wireless body-areanetwork," IEEE J. Solid-State Circuits, vol. 47, no. 11, pp. 2678-2692, Nov. 2012 .

[3] M. H. Seyedi, B. Kibret, D. Lai, and M. Faulkner, "A survey on intrabody communications for body area network applications," IEEE Trans. Biomed. Eng., vol. 60, no. 8, pp. 2067-2079, Aug. 2013.

[4] M. S. Wegmueller, M. Hediger, T. Kaufmann, F. Buergin, and W. Fichtner, "Wireless implant communications for biomedical monitoring Sensor network," in Proc., IEEE ISCAS 2007, Orleans, USA, May 2007, pp. 809-812.

[5] Y. T. Lin, Y. S. Lin, C. H. Chen, H. S. Chen, Y. C. Yang, and S. S. Lu, "A $0.5-\mathrm{V}$ biomedical system-on-a-chip for intrabody communication system," IEEE Trans. Ind. Electron., vol. 58, no. 2, pp. 690-699, Feb. 2011.

[6] T. G. Zimmerman, "Personal area networks (PAN): Near-field intrabody communication," M.S. thesis, Media Lab., Massachusetts Inst Technol., Cambridge, MA, USA, 1995.

[7] Z. Lucev, I. Krois, and M. Cifrek, "A multichannel wireless EMG measurement system based on intrabody communication," in Proc., IMEKO World Congress, Portugal, Sep. 2009, pp. 1711-1715.

[8] M. S. Wegmueller, M. Oberle, N. Felber, N. Kuster, and W. Fichtner, "Signal transmission by galvanic coupling through the human body," IEEE Trans. Instrum. Meas., vol. 59, no. 4, pp. 963-969, Apr. 2010.

[9] C. Hyoung, J. Song, J. Hwang, J, Kim, D. Park, and S. Kang, “A novel system for intrabody communicatoin: touch-and-plat," in Proc., IEEE ISCAS 2006, Greece, May 2006, pp. 1343-1346.

[10] P. Harikumar, M. Kazim, and J. Wikner, "An analog receiver front-end for capacitive body-coupled communcation," in Proc., 30rd IEEE NORCHIP 2012, Denmark, pp. 1-4.

[11] A. N. Kim, P. A. Floor, T. A. Ramstad, and I. Balasingham "Communication using ultra wide-band pulse position modulation for in-body sensors," in Proc., 7th IEEE/ACM Body Area Networks Conference (Bodynets) 2012, Norway, pp. 159-165.

[12] M. Hämäläinen, Singleband UWB systems: analysis and measurements of coexistence with selected existing radio systems, University of Oulu, 2006.

[13] International Commission on Non-Ionizing Radiation Protection (ICNIRP), "Guidelines for limiting exposure to time-varying electric, magnetic, and electromagnetic fields (up to $300 \mathrm{GHz}$ )," Health Physics Society, vol 74, no. 4, pp. 494-522, 1998.

[14] L. Ge, G. Yue, and S. Affes, "On the BER performance of pulseposition-modulation UWB radio in multipath channels," in Proc., IEEE Conf. Ultra Wideband Systems Technologies, Baltimore, MD, May 2002, pp. 231-234. 